\title{
Anterior cruciate ligament reconstruction: how do we perform it? Brazilian orthopedic surgeons' preference
}

\author{
Luiz Felipe Morlin Ambra ${ }^{1}$ - Fernando Cury Rezende ${ }^{1}$ Bruno Xavier ${ }^{1}$. \\ Felipe Conrado Shumaker ${ }^{1}$. Carlos Eduardo da Silveira Franciozi ${ }^{1}$. \\ Marcos Vinicius Malheiros Luzo ${ }^{1}$
}

Received: 27 April 2015 / Accepted: 24 June 2015 / Published online: 15 July 2015

(C) SICOT aisbl 2015

\begin{abstract}
Purpose We evaluated current trends and common practice of Brazilian orthopedic surgeons while selecting approaches for anterior cruciate ligament (ACL) reconstruction surgery.

Methods Orthopedic surgeons $(n=191)$ completed a survey consisting of seven questions regarding their profiles and preference for ACL reconstruction techniques.

Results Most surgeons were from Southeast Brazil (56.6 \%) and had specialized in knee surgery (79.5\%); most participants $(55.1 \%)$ had worked in this field for $>$ five years, and $46.8 \%$ had performed $>50$ ACL reconstructions. Further, $93.1 \%$ respondents preferred the hamstring graft. Analysis of preference for the femoral tunnel approach in terms of years of experience showed that surgeons with ten to 15 years' experience preferred the transtibial approach; those with $<$ five years of experience, the transportal technique; those with $>15$ years' experience, the two-incision technique.

Conclusions Surgeons' preferences for ACL reconstruction are variable, and are influenced by learning time and availability of tools rather than research evidence.
\end{abstract}

Keywords Anterior cruciate ligament · Surgeons · Orthopedic surgeons $\cdot$ Surgical approach

Luiz Felipe Morlin Ambra felipeambra71@gmail.com

1 Departamento de Ortopedia e Traumatologia, da Universidade Federal de São Paulo - Escola Paulista de Medicina (DOT - Unifesp/ EPM), Rua Caraíbas, 666 Apto 101 Perdizes, CEP 05020-000 São Paulo, SP, Brazil

\section{Introduction}

Anterior cruciate ligament (ACL) injuries are among the most common knee ligament injuries in the world. Consequently, it is one of the most extensively studied orthopedic conditions, with more than 1,100 manuscripts published in 2013 [1]. The research regarding ACL reconstruction has focused on individual practice patterns, choice of graft type, surgical approach, and other technical aspects of the surgical procedure.

The therapeutic goals of ACL reconstruction are to restore stability of the knee joint, prevent secondary lesions to other structures, and improve functional outcomes. The evolution of surgical technology has provided the surgeon with choice on the types of grafts, fixation devices, and surgical techniques to reach these clinical outcomes [2]. Guidelines for the surgical management of ACL injuries are based on scientific findings rather than on expert opinion. Prospective randomized clinical trials (RCTs) are considered to provide the best quality of evidence in the medical literature. RCTs are the source of data for systematic reviews and meta-analyses, together comprising level-1 evidence that guide clinical decision-making. Evidence-based medicine has evolved from an outpost of clinical research to the mainstream of clinical practice [3]. Adherence to evidence-based medicine is not always possible, particularly in areas where high-quality evidence is not available or where evidence is inconclusive [3]. Thus, the absence of clear evidence that supports clinical evidence can lead to inconsistencies between surgeons' clinical practice and trends and recommendations in the research literature [4].

The aims of this study were to evaluate current trends and common practice of Brazilian orthopedic surgeons while selecting approaches for ACL reconstruction surgery. The primary goal of our investigation was to understand surgeons' preferences and opinions on this surgery. The second goal was 
Table 1 Region of origin of the study participant

\begin{tabular}{llllll}
\hline & Southeast & Northeast & South & West Center & North \\
\hline State distribution (\%) & 56.6 & 20.8 & 12 & 5.9 & 4.7 \\
\hline
\end{tabular}

to compare the opinions of Brazilian orthopedic surgeons with the most recent published level-1 evidence.

\section{Materials and methods}

\section{Study subjects}

Before commencement, this study was approval by the Federal University of São Paulo Research Ethics Board.

a

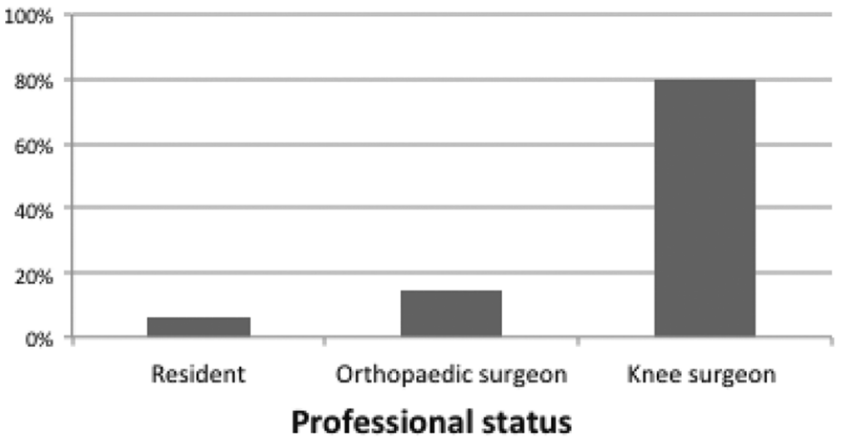

C

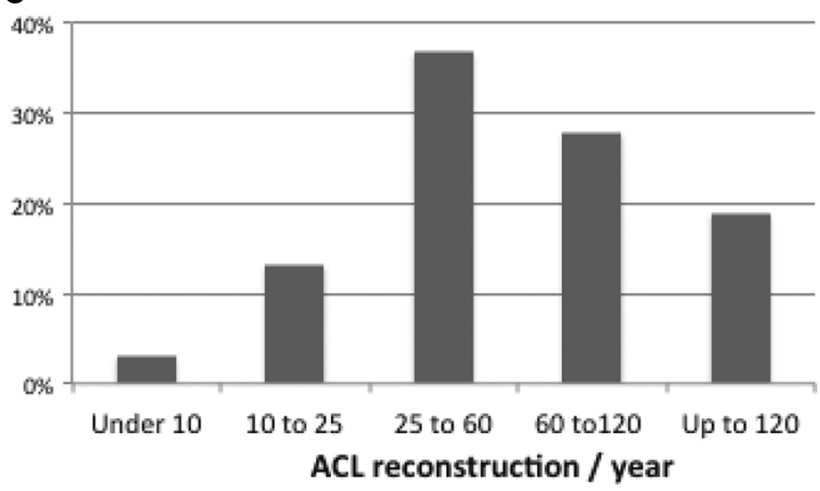

\section{Survey design}

The current survey comprised seven questions regarding ACL reconstruction. The first question addressed professional status (i.e., specialist surgeon, fellow, or resident). The second queried about the number of years of experience. The third question was regarding the number of ACL reconstructions surgeons performed in the last year. The fourth question was on the surgical approach of drilling the femoral tunnel (anteromedial arthroscopic portal [AMP], arthroscopic

b

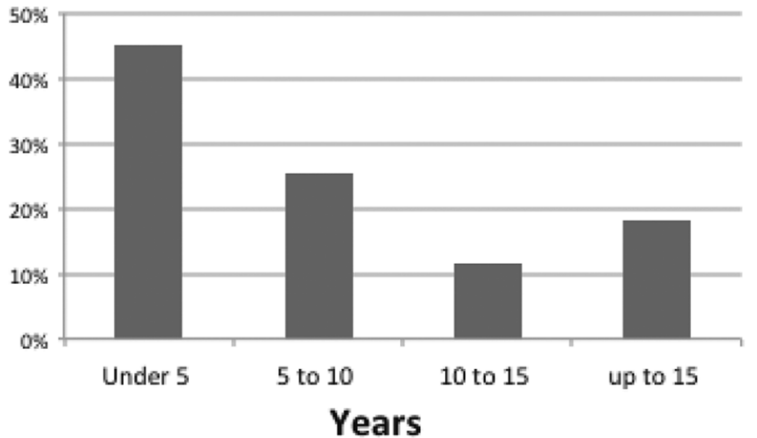

d

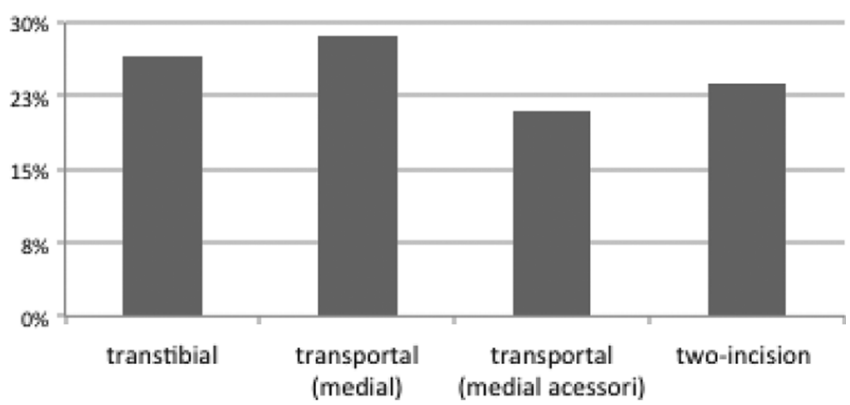

Femoral Tunnel technique

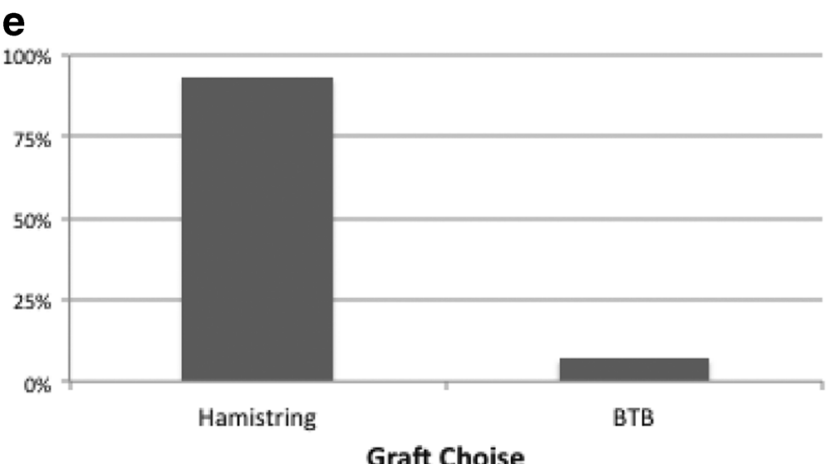

Fig. 1 Survey results. a Professional status of participants. b Surgeons' experience, in years, for ACL reconstruction. c Frequency of ACL reconstructions surgeon perform per year. $\mathbf{d}$ Technique preferred to perform femoral tunnel. e Preferred graft used for ACL reconstruction 


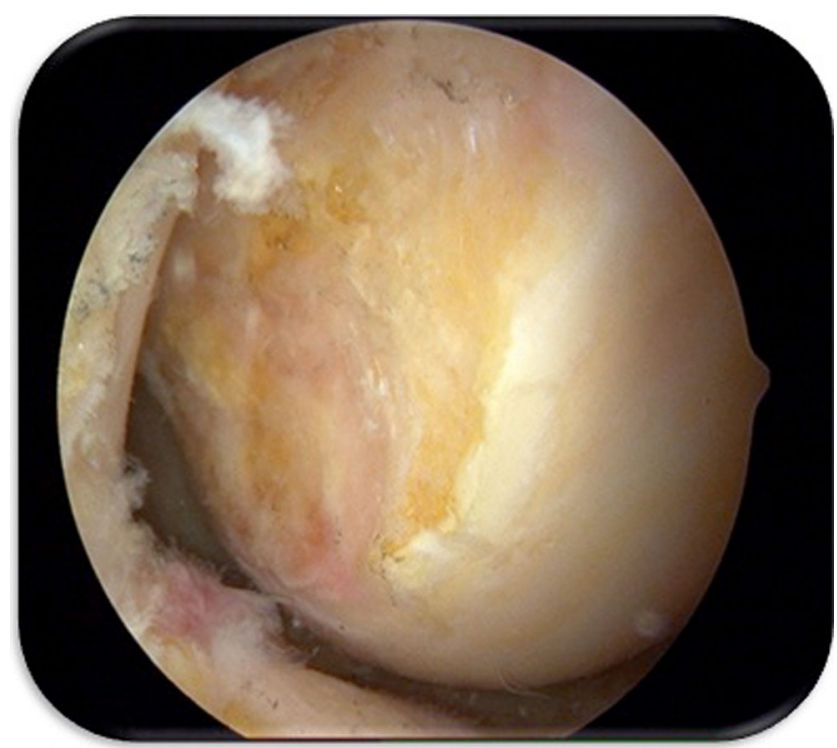

Fig. 2 Arthroscopy view of the whole medial wall of lateral condyle

transtibial, or two-incision techniques). The fifth question was about the preferred graft (patellar tendon, hamstring tendon, quadriceps tendon or allograft). The responders were asked to provide their city of origin. After completing the survey, the interviewer showed an arthroscopic image of the medial wall of the lateral condyle from the anterior medial portal, and the participants were asked to mark the anatomic position of the femoral tunnel during single-band ACL reconstruction.

\section{Survey distribution}

The survey was performed on the 15 th edition of Brazilian Congress of Knee Surgery, held on April 10-12,

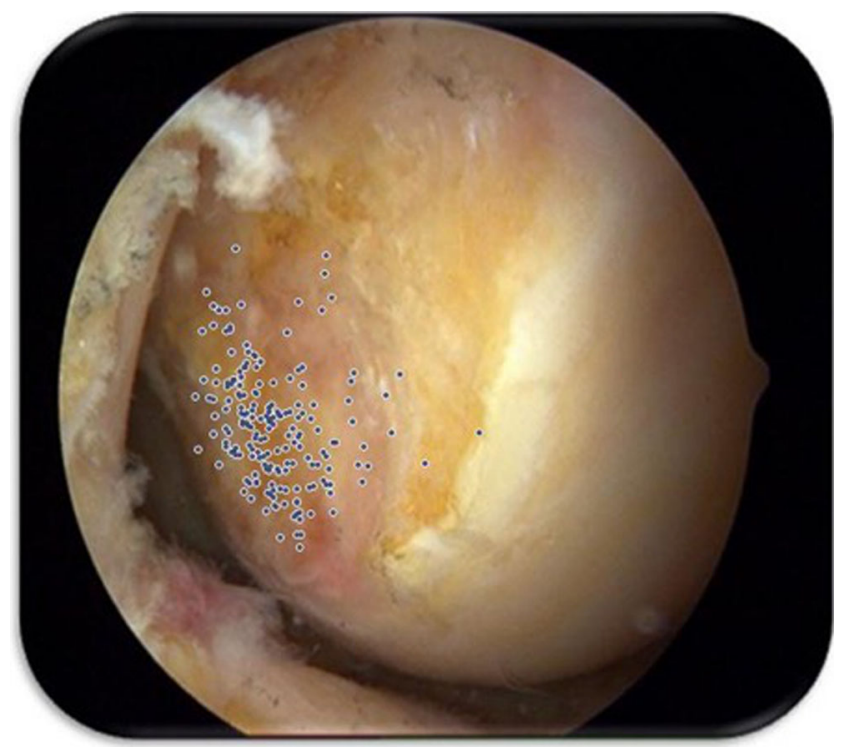

Fig. 3 All surgeons' positions of the femoral tunnel

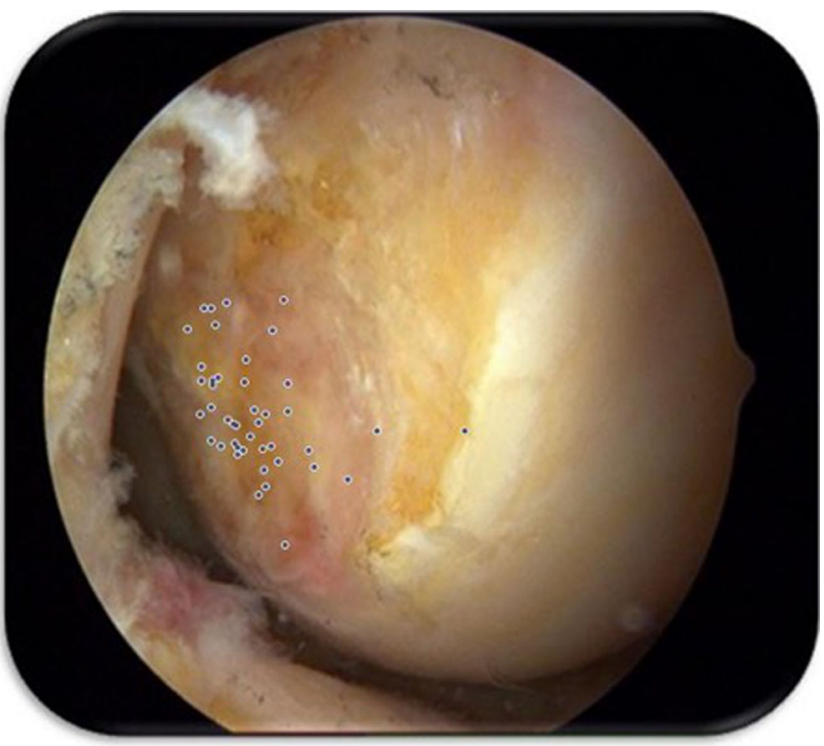

Fig. 4 Outside-in technique

2014, in Maceio (AL). The questionnaire was administered to surgeons while entering the theatre for the presentation and was collected after surgeons completed the questionnaire. One volunteer was always present to resolve any doubt about the survey at the site were the questionnaire was provided.

\section{Data analysis}

Frequency distributions were determined for all the questions, and the $z$-test (two equal proportion test) was applied. The chisquared test was chosen to analyze the association between categories. Significance level for all comparisons was $P<0.05$.

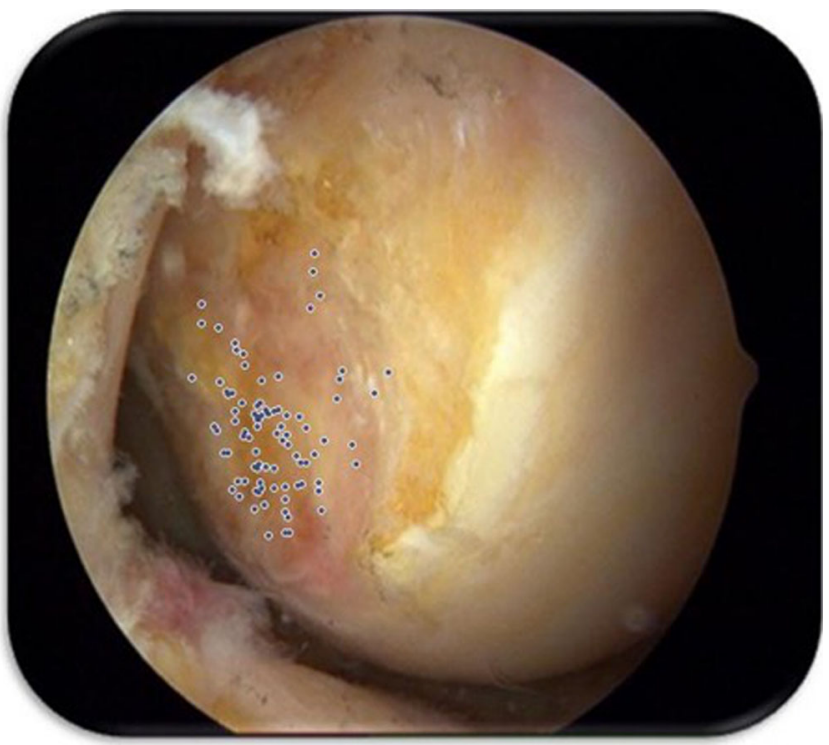

Fig. 5 Transportal technique 


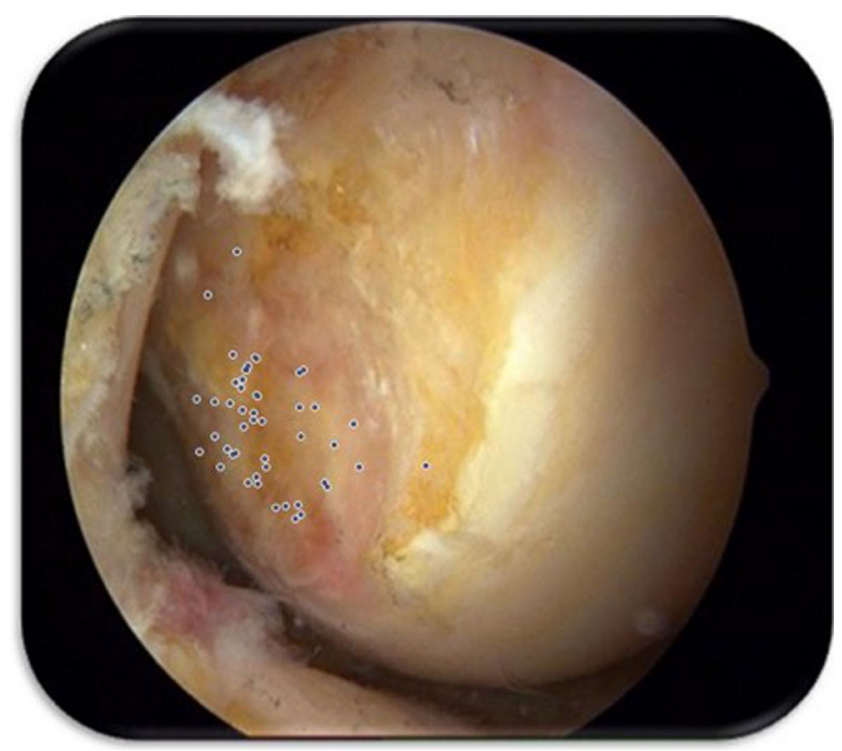

Fig. 6 Transtibial technique

\section{Results}

During the 15th edition of Brazilian Congress of Knee Surgery, 191 surgeons completed the questionnaire on surgical practices about ACL reconstruction.

Most surgeons were from the southeast, including $56.6 \%$ of participants (Table 1).

The initial objective of the survey was to determine the surgeon's background and to analyze the duration of experience, academic training, and surgical practice. The first question evaluated surgeon's personal status; most had graduated as knee surgeons (79.5\%). The second question was related to number of years of surgeons' experience, and most of the participants $(55.1 \%)$ had more than five years' experience in this field. With respect the surgeon's practice, $46.8 \%$ had performed $>50$ ACL reconstructions in the last year. The results are shown in Fig. 1.

Most participants $(93.1 \%)$ preferred the hamstring as the standard graft in primary ACL reconstruction.

The fifth question queried surgeons about their preferences while performing femoral tunnel during ACL reconstruction, and $49.7 \%$ surgeons who completed the survey preferred the transportal technique. However, while evaluating preference for femoral tunnel with regard to years of experience, we

Table 2 Relation between femoral tunnel and time of experience, in years

\begin{tabular}{lllll}
\hline Years of experience & Transtibial & Transportal & Two incisions & Total \\
\hline Under 5 & 14 & 27 & 17 & 58 \\
$5-10$ & 11 & 19 & 3 & 33 \\
$10-15$ & 2 & 10 & 3 & 15 \\
Over 15 & 10 & 0 & 16 & 26 \\
\hline
\end{tabular}

found that the transtibial technique was most used by surgeons with ten to 15 years' experience, the transportal technique was preferred by those having < five years' experience, and the two-incision technique was preferred by who those having $>15$ years' experience.

The last part of the survey evaluated the femoral tunnel position in the anatomical reconstruction of the ACL. We provided a picture of the whole medial wall of the lateral femoral condyle (Fig. 2). The picture was taken from the arthroscopy with the camera positioned in the anterior medial portal after a proper cleaning of the ACL insertion site. Then, the surgeons were asked to mark in the picture the exact point where they considered the anatomical position to perform the femoral tunnel in ACL reconstruction. The answers for this question vary greatly as shown in Fig. 3; however, there was no relationship between the answers obtained (position of the femoral tunnel entry point) and the technique used (Figs. 4, 5, and 6).

\section{Discussion}

The present survey provides information from 190 orthopedic surgeons who had participated in the Brazilian Congress of Knee Surgery, 2014; most of these participants (79.5\%) were knee surgeons, $14.7 \%$ were not knee surgeons, and $5.8 \%$ were residents. Most participants were young surgeons (44.9\% with less than 5 years' experience) and more than half were less experienced knee surgeons, as $53.2 \%$ perform few ACL reconstructions per month (up to five operations).

The purpose of this study was to compare data from Brazilian knee surgery practitioners with those of other worldwide literature surveys, including some controversial topics of ACL surgery such as graft options, femoral tunnel choices (anatomic and transtibial techniques), as well as an overview of the Brazilian knee surgeon's understanding of an ideal anatomic location of the femoral tunnel in ACL reconstruction.

\section{Graft choice}

One of the main findings of the survey was that most (93.1\%) knee surgeons performed ACL reconstructions using hamstring grafts, and only $6.9 \%$ of them preferred the bonepatellar tendon bone (BPTB) grafts. Although these results are consistent with those of the worldwide trend of preference for hamstrings grafts over BPTB grafts, previous similar reports from other countries have not shown large differences in preference [4]. In contrast to this current trend, the literature does not support the use of hamstring grafts over BPTB grafts. With respect to knee stability after ACL surgery, BPTB grafts have shown to provide superior rotational control, as demonstrated by a better pivot shift control [5]. Besides, lower postoperative failure rates have been reported in previous meta- 
analyses that compared these two graft options [6, 7]. However, the reasons why hamstring grafts are more popular may be related to the lower post-operative incidence of anterior knee pain and extension deficits, as well as a higher patient acceptance due to better cosmetic results [8].

\section{Femoral tunnel}

In the last two decades, there has been a growing interest in the anatomical placement of the femoral tunnel during ACL reconstruction. Although not well established in the literature, isometric or transtibial location of femoral tunnels did not result in adequate rotational control, as demonstrated by biomechanical studies [9]. The femoral tunnel may not be drilled independently from the tibial tunnel in transtibial techniques, and therefore, does not guarantee the anatomical location of the femoral tunnel $[10,11]$. On the other hand, independent drilling procedures, such as transportal and two-incision techniques, allow the surgeon to pursue a wider range of freedom for femoral tunnel placement, thereby reducing the risk of an inadvertent tunnel location $[11,12]$. The present survey shows growing interest in anatomical ACL reconstruction, since only $26.5 \%$ of Brazilian surgeons still perform a trans-tibial or isometric procedure. Advocates of independent drilling techniques correspond to $72.5 \%$ of ACL surgeries in this survey, $49.7 \%$ of these prefer transportal and $23.8 \%$ prefer twoincision (outside-in) techniques. These results reflect the current trend of changing focus of performing femoral tunnels from a more vertical location to a more horizontal location, which is consistent with a recent worldwide survey [1]. However, such change in direction in femoral tunnel placement may be based only on a surgeon's impression of superior results with anatomical ACL reconstruction, as it is still not supported by clinical data [11]. Although current evidence has shown better knee stability with independent (anatomical) drilling techniques for ACL reconstruction, clinical results between transtibial and anatomical ACL reconstruction techniques were not different [12]. Further trials should be performed to better resolve this controversy, as newer ACL techniques are predominantly based on the anatomical placement of femoral tunnels.

Another aim of our study finding was whether a surgeon's judgment of a femoral tunnel's location really represents its approximate ideal location. An arthroscopic view of the medial wall of the lateral condyle was used to accomplish this task, and we obtained interesting findings. Many surgeons did not have the knowledge of a well-positioned femoral tunnel, as noted from the variability in the anatomical locations selected by surgeons. This is concerning because, despite surgeons' preference for the femoral tunnel technique (either anatomical or transtibial), many knee surgeons are not familiar with femoral tunnel landmarks, which may compromise post- operative outcomes, as the femoral tunnel location is critical for the success of ACL surgeries.

Other remarkable finding from this survey was the choice of the surgery technique among experienced knee surgeons. In the present survey, although the transportal technique was described as the most popular technique among the surgeons, none of the surgeons with more than 15 years of experience preferred this procedure. The outside-in technique was preferred by the majority experienced surgeons $(41 \%)$ and $27 \%$ used the trans-tibial technique (Table 2). This choice may be related to the origins of ACL reconstruction surgery, as reported in the literature since the 1980s [2].

Surgeon preferences for the method of ACL reconstruction may vary. The choices can be motivated by learning time or availability of tools. However, the reason why a method is commonly used may not always be supported by evidencebased publications.

Conflict of interest The authors declare that they have no competing interests.

\section{References}

1. Mall NA, Chalmers PN, Moric M, Tanaka MJ, Cole BJ, Bach BR Jr, Paletta GA Jr (2014) Incidence and trends of anterior cruciate ligament reconstruction in the United States. Am J Sports Med 42: 2363-2370. doi:10.1177/0363546514542796

2. Samuelsson K, Andersson D, Ahlden M, Fu FH, Musahl V, Karlsson J (2013) Trends in surgeon preferences on anterior cruciate ligament reconstructive techniques. Clin Sports Med 32:111126. doi:10.1016/j.csm.2012.08.011

3. Suk M, Hanson B, Helfet DL (2010) Evidence-based orthopedic surgery: is it possible? Orthop Clin North Am 41:139-143. doi:10. 1016/j.ocl.2009.11.002

4. Chechik O, Amar E, Khashan M, Lador R, Eyal G, Gold A (2013) An international survey on anterior cruciate ligament reconstruction practices. Int Orthop 37:201-206. doi:10.1007/s00264-012-1611-9

5. Biau DJ, Katsahian S, Kartus J, Harilainen A, Feller JA, Sajovic M, Ejerhed L, Zaffagnini S, Ropke M, Nizard R (2009) Patellar tendon versus hamstring tendon autografts for reconstructing the anterior cruciate ligament: a meta-analysis based on individual patient data. Am J Sports Med 37:2470-2478. doi:10.1177/0363546509333006

6. Persson A, Fjeldsgaard K, Gjertsen JE, Kjellsen AB, Engebretsen L, Hole RM, Fevang JM (2014) Increased risk of revision with hamstring tendon grafts compared with patellar tendon grafts after anterior cruciate ligament reconstruction: a study of 12,643 patients from the Norwegian Cruciate Ligament Registry, 2004-2012. Am J Sports Med 42:285-291. doi:10.1177/0363546513511419

7. Reinhardt KR, Hetsroni I, Marx RG (2010) Graft selection for anterior cruciate ligament reconstruction: a level I systematic review comparing failure rates and functional outcomes. Orthop Clin North Am 41:249-262. doi:10.1016/j.ocl.2009.12.009

8. Sadoghi P, Muller PE, Jansson V, van Griensven M, Kropfl A, Fischmeister MF (2011) Reconstruction of the anterior cruciate ligament: a clinical comparison of bone-patellar tendon-bone single bundle versus semitendinosus and gracilis double bundle technique. Int Orthop 35:127-133. doi:10.1007/s00264-010-1037-1 
9. Gavriilidis I, Motsis EK, Pakos EE, Georgoulis AD, Mitsionis G, Xenakis TA (2008) Transtibial versus anteromedial portal of the femoral tunnel in ACL reconstruction: a cadaveric study. Knee 15:364-367. doi:10. 1016/j.knee.2008.05.004

10. Alentorn-Geli E, Lajara F, Samitier G, Cugat R (2010) The transtibial versus the anteromedial portal technique in the arthroscopic bone-patellar tendon-bone anterior cruciate ligament reconstruction. Knee Surg Sports Traumatol Arthrosc 18:1013-1037. doi:10.1007/s00167-009-0964-0
11. Bedi A, Musahl V, Steuber V, KendoffD, Choi D, Allen AA, Pearle AD, Altchek DW (2011) Transtibial versus anteromedial portal reaming in anterior cruciate ligament reconstruction: an anatomic and biomechanical evaluation of surgical technique. Arthroscopy 27:380-390. doi:10.1016/j.arthro.2010.07.018

12. Riboh JC, Hasselblad V, Godin JA, Mather RC 3rd (2013) Transtibial versus independent drilling techniques for anterior cruciate ligament reconstruction: a systematic review, meta-analysis, and meta-regression. Am J Sports Med 41:2693-2702. doi:10. $1177 / 0363546513506979$ 